\title{
Thermal Conductivity of the One-Dimensional Fermi-Hubbard Model
}

\author{
C. Karrasch, ${ }^{1,2,5}$ D. M. Kennes, ${ }^{3}$ and F. Heidrich-Meisner ${ }^{4}$ \\ ${ }^{1}$ Department of Physics, University of California, Berkeley, California 95720, USA \\ ${ }^{2}$ Materials Sciences Division, Lawrence Berkeley National Laboratory, Berkeley, California 94720, USA \\ ${ }^{3}$ Institut für Theorie der Statistischen Physik, RWTH Aachen University and JARA-Fundamentals of Future Information Technology, \\ 52056 Aachen, Germany \\ ${ }^{4}$ Department of Physics and Arnold Sommerfeld Center for Theoretical Physics, Ludwig-Maximilians-Universität München, \\ 80333 München, Germany \\ ${ }^{5}$ Dahlem Center for Complex Quantum Systems and Fachbereich Physik, Freie Universität Berlin, 14195 Berlin, Germany
}

(Received 18 June 2015; published 9 September 2016)

\begin{abstract}
We study the thermal conductivity of the one-dimensional Fermi-Hubbard model at a finite temperature using a density matrix renormalization group approach. The integrability of this model gives rise to ballistic thermal transport. We calculate the temperature dependence of the thermal Drude weight at half filling for various interaction strengths. The finite-frequency contributions originating from the fact that the energy current is not a conserved quantity are investigated as well. We report evidence that breaking the integrability through a nearest-neighbor interaction leads to vanishing Drude weights and diffusive energy transport. Moreover, we demonstrate that energy spreads ballistically in local quenches with initially inhomogeneous energy density profiles in the integrable case. We discuss the relevance of our results for thermalization in ultracold quantum-gas experiments and for transport measurements with quasi-onedimensional materials.
\end{abstract}

DOI: 10.1103/PhysRevLett.117.116401

Improving our understanding of transport in onedimensional (1D) strongly correlated systems (SCSs) is an active field in condensed matter theory. While in 1D the existence of powerful numerical [1-3] and analytical [4,5] methods makes it possible to obtain quantitative results (see, e.g., [4,6-8]), transport coefficients are very hard to come by exactly and are challenging quantities to determine numerically. Early studies suggested that integrable systems such as the 1D spin-1/2 Heisenberg or FermiHubbard model (FHM) may possess ballistic transport properties at finite temperatures [9]. In the linear response theory, ballistic dynamics manifests itself through nonzero Drude weights. The so far best understood model is the spin-1/2 $X X Z$ chain, for which the Drude weight for thermal transport has been calculated exactly $[10,11]$, while substantial progress has recently been made regarding the spin conductivity [12-21].

The theory of transport in the 1D FHM is much less advanced and has focused on spin and charge transport [22-28]. The thermal conductivity can, by using the Kubo formula $[29,30]$, be written as

$$
\operatorname{Re} \kappa(\omega)=2 \pi D_{\text {th }}(T) \delta(\omega)+\kappa_{\text {reg }}(\omega)
$$

with the thermal Drude weight $D_{\text {th }}(T)$ and a regular finite-frequency contribution $\kappa_{\text {reg }}(\omega)$. Since in SCSs the Wiedemann-Franz law is not necessarily valid, independent calculations of charge and thermal transport are required.
The formal argument to prove a nonzero Drude weight relies on the Mazur inequality $[9,31,32]$

$$
D_{\text {th }} \geq \frac{1}{2 T^{2} L} \sum_{i} \frac{\left\langle Q_{i} I_{\mathrm{th}}\right\rangle^{2}}{\left\langle Q_{i}^{2}\right\rangle},
$$

where $I_{\mathrm{th}}$ is the energy-current operator and the $Q_{i}$ are local or quasilocal conserved quantities $[12,15,33]$. In the presence of interactions, nontrivial $Q_{i}$ leading to finite Drude weights typically exist in integrable models [9]. For instance, for the spin-1/2XXZ chain, the energy current $I_{\text {th }}=Q_{3}$ itself is conserved [implying that $\kappa_{\text {reg }}(\omega)=0$ ], while for the FHM, $I_{\text {th }}$ has only a partial overlap with $Q_{3}$ (both operators have a similar structure [34]) such that while $D_{\text {th }}>0$, also $\kappa_{\text {reg }}(\omega) \neq 0$ [9]. As a consequence, the half-filled FHM realizes an unusual behavior: ballistic thermal transport [9], yet diffusive charge conduction $[27,28]$ at temperatures $T>0$.

Here, we address the outstanding problem of quantitatively calculating $\operatorname{Re} \kappa(\omega)$ at $T>0$ for the FHM at half filling by using a finite-temperature density matrix renormalization group (DMRG) method [3,47-51], previously applied to both charge transport in the FHM [27] and transport in quasi-1D spin-1/2 systems [14,21,47,52,53]. We obtain the energy-current autocorrelation function $C_{\text {th }}(t)=\operatorname{Re}\left\langle I_{\text {th }}(t) I_{\text {th }}\right\rangle / L$ from time-dependent simulations. Since $C_{\text {th }}(t)$ saturates fast at a time-independent nonzero value, we are able to extract (i) the thermal Drude weight and (ii) the regular part from a Fourier transformation in combination with a linear prediction [54]. Moreover, 
we consider the extended FHM as an example for a nonintegrable model and provide evidence that ballistic contributions are absent, with a diffusive form of the low-frequency $\kappa_{\text {reg }}(\omega)$.

The FHM has been realized with ultracold quantum gases [55-64]. In ultracold quantum gases, relaxation processes play an important role for reaching thermal equilibrium during the state preparation $[65,66]$, and thermometry is an open experimental problem [67]. Furthermore, understanding thermalization dynamics and nonequilibrium transport as such have been the goal of several optical-lattice experiments with Hubbard systems [68-71]. We demonstrate that real-space perturbations in the energy density spread ballistically in the 1D FHM at $T>0$ while charge diffuses $[27,28]$, providing a route to experimentally observing the qualitative difference between charge and energy dynamics in this model.

Definitions. - The Hamiltonian of the extended FHM is given by $H=\sum_{l=1}^{L-1} h_{l}$ with local terms

$$
\begin{aligned}
h_{l}= & -t_{0} \sum_{\sigma}\left(c_{l \sigma}^{\dagger} c_{l+1 \sigma}+\text { H.c. }\right)+V\left(n_{l}-1\right)\left(n_{l+1}-1\right) \\
& +\frac{U}{2}\left[\left(n_{l \uparrow}-\frac{1}{2}\right)\left(n_{l \downarrow}-\frac{1}{2}\right)\right. \\
& \left.+\left(n_{l+1 \uparrow}-\frac{1}{2}\right)\left(n_{l+1 \downarrow}-\frac{1}{2}\right)\right],
\end{aligned}
$$

where $c_{l \sigma}$ annihilates a fermion with spin $\sigma$ on site $l$ and $n_{l \sigma}=c_{l \sigma}^{\dagger} c_{l \sigma} . U$ and $V$ denote the on-site and the nearestneighbor Coulomb repulsion, respectively. We use open boundary conditions. All results in the main text are for half filling $n=N / L=1$, where $N$ is the total number of fermions. For convenience, we implemented the FHM as a two-leg spin-1/2 ladder [34].

We derive the energy current from the continuity equation [9], leading to $I_{\text {th }}=i \sum_{l=1}^{L-2}\left[h_{l}, h_{l+1}\right]$ (for the full expression, see [34]). At $n=1$, particle-hole symmetry leads to a vanishing thermopower [34,72], and, thus, the thermal conductivity stems solely from energy-current correlations.

Numerical method. - The thermal Drude weight is related to the long-time asymptote of the current correlation functions via

$$
D_{\text {th }}=\lim _{t \rightarrow \infty} \lim _{L \rightarrow \infty} \frac{C_{\text {th }}(t)}{2 T^{2}},
$$

and the regular part of the conductivity defined in Eq. (1) can be obtained from $\left[\tilde{C}_{\mathrm{th}}(t)=C_{\mathrm{th}}(t)-2 T^{2} D_{\mathrm{th}}\right]$

$$
\kappa_{\mathrm{reg}}(\omega)=\frac{1-e^{-\omega / T}}{\omega T} \operatorname{Re} \int_{0}^{\infty} d t e^{i \omega t} \lim _{L \rightarrow \infty} \tilde{C}_{\mathrm{th}}(t) .
$$

Note that the derivation of the Kubo formula for $\kappa$ is more subtle than for the charge conductivity (see, e.g., Refs. [30,73-77], and references therein, and Ref. [34] for a discussion), while there is also ongoing research on thermal and energy transport in an open quantum system (see, e.g., [78-81]).

Our finite- $T$ DMRG method, implemented via matrixproduct states [82-85], is based on the purification trick [86] (see [49,54,87-89] for related work). Thus, we simulate pure states that live in a Hilbert space spanned by the physical and auxiliary (ancilla) degrees of freedom. Mixed states are obtained by tracing over the ancillas. In order to access time scales as large as possible, we employ a finite-temperature disentangler [47], using that purification is not unique to slow down the entanglement growth. Moreover, we exploit "time-translation invariance" [49], rewrite $\left\langle I_{\mathrm{th}}(t) I_{\mathrm{th}}(0)\right\rangle=\left\langle I_{\mathrm{th}}(t / 2) I_{\mathrm{th}}(-t / 2)\right\rangle$, and carry out two independent calculations for $I_{\mathrm{th}}(t / 2)$ as well as for $I_{\mathrm{th}}(-t / 2)$. Since the energy current is a six-point function, a tDMRG simulation for the energy-current autocorrelation is much more demanding than it is in the charge case. Our calculations are performed with $L=100$ sites (see Fig. S1 in Ref. [34] for an analysis of the $L$ dependence). The "finite-time" error of $\kappa_{\text {reg }}(\omega)$ can be assessed following Ref. [53], resulting in the error bars shown in the figures.

Real-time decay of $C_{\mathrm{th}}$.-Typical DMRG results for $C_{\text {th }}(t)$ are shown in Figs. 1(a) and 1(b) for $U=2 t_{0}$ and $U=8 t_{0}$, respectively, and temperatures $T=\infty, 2 t_{0}, 2 t_{0} / 3$. We are able to reach times $t t_{0} \lesssim 5$. For $V=0$ (thick lines), i.e., in the integrable case, $C_{\text {th }}(t)$ rapidly saturates at a constant nonzero value, reflecting the ballistic nature of energy transport in this model. The transients are surprisingly short compared to spin transport in the spin- $1 / 2 \mathrm{XXZ}$ chain [14] and exhibit oscillations with a fairly small amplitude. To illustrate the behavior in the nonintegrable extended FHM, we present data for $V=U / 3$ (thin lines), for which our system Eq. (3) is still in the Mott-insulating phase [90]. The DMRG results unveil a much stronger

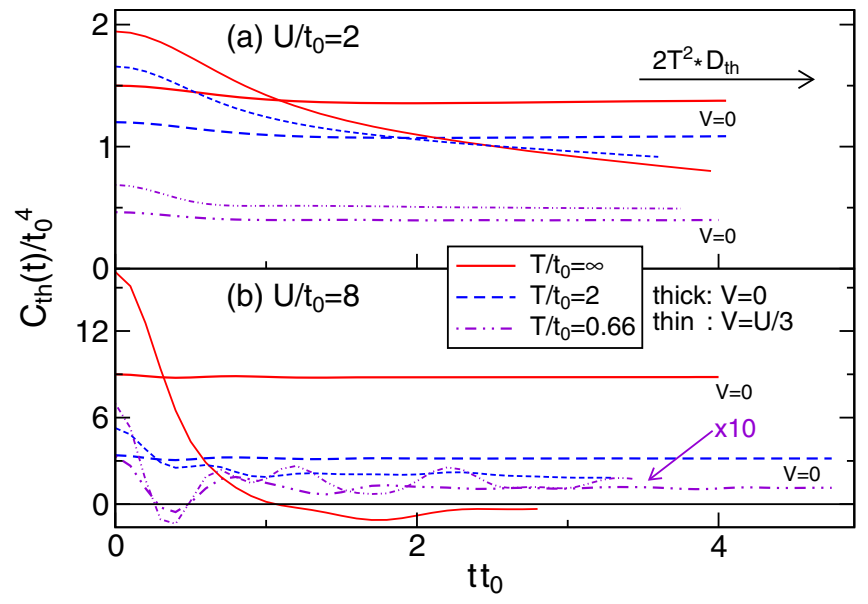

FIG. 1. DMRG results for $C_{\mathrm{th}}(t)$ for various $T>0$ at (a) $U / t_{0}=2$ and (b) $U / t_{0}=8$. Thick lines, $V=0$; thin lines, $V=U / 3$. The curves for $U / t_{0}=8$ and $T / t_{0}=0.66$ are multiplied by a factor of 10 . 
decay of $C_{\mathrm{th}}(t)$ compared to the integrable case yet also longer transient dynamics before the asymptotic regime is reached [see, e.g., the data for $T=\infty$ shown in Fig. 1(a)]. For $U / t_{0}=8$ and $T=\infty$, the real-time decay of $C_{\mathrm{th}}$ is consistent with a vanishing $D_{\text {th }}(T)$, as expected for this nonintegrable model [53,91-94].

Thermal Drude weight for $V=0$. - The fast saturation of $C_{\mathrm{th}}(t)$ at a constant and nonzero value allows us to extract the temperature dependence of $D_{\mathrm{th}}(T)$, displayed in Fig. 2 for $U / t_{0}=0,1,2,4,8$ (note the log-log scale). For $U=0$, we compare our data to the exact result

$$
D_{\mathrm{th}}(T)=\frac{t_{0}^{2}}{2 \pi T^{2}} \int_{-\pi}^{\pi}\left[\epsilon_{k} v_{k} f\left(\epsilon_{k}\right)\right]^{2} e^{\epsilon_{k} / T} d k,
$$

where $\epsilon_{k}=-2 t_{0} \cos (k), \quad v_{k}=\partial \epsilon_{k} / \partial k, \quad$ and $\quad f(\epsilon)=$ $1 /\left(1+e^{\epsilon / T}\right)$. The agreement is excellent. In general, $D_{\text {th }}(T)$ has a maximum at a $U$-dependent temperature that shifts to a larger temperature as $U$ increases. In the hightemperature regime $T>t_{0}, D_{\text {th }}=D_{\text {th }}^{\infty} / T^{2}$ (dashed lines in the figure), where the prefactor $D_{\mathrm{th}}^{\infty}$ has been extracted from the numerical data at $\beta=0$. In the Supplemental Material [34], we compare the thermal Drude weight of the FHM to the one of the Heisenberg chain [10]. The latter describes the low-temperature contribution of spin excitations to the full $D_{\text {th }}$ of the FHM for $U \gg t_{0}$ at $n=1$, while we present results for the spin-incoherent regime $T \gg 4 t_{0}^{2} / U$, where charge excitations dominate.

We next study how much of the full spectral weight of Re $\kappa(\omega)$ is in the Drude peak by plotting $2 \pi D_{\text {th }} / I_{0}$ versus $U / t_{0}$ at $\beta=0$ in the inset in Fig. 2 , where $I_{0}=\int d \omega \operatorname{Re} \kappa(\omega)$. The Drude weight contains the full weight $I_{0}$ only at $U=0$ and for $U / t_{0} \rightarrow \infty$. In the former case, this results from the

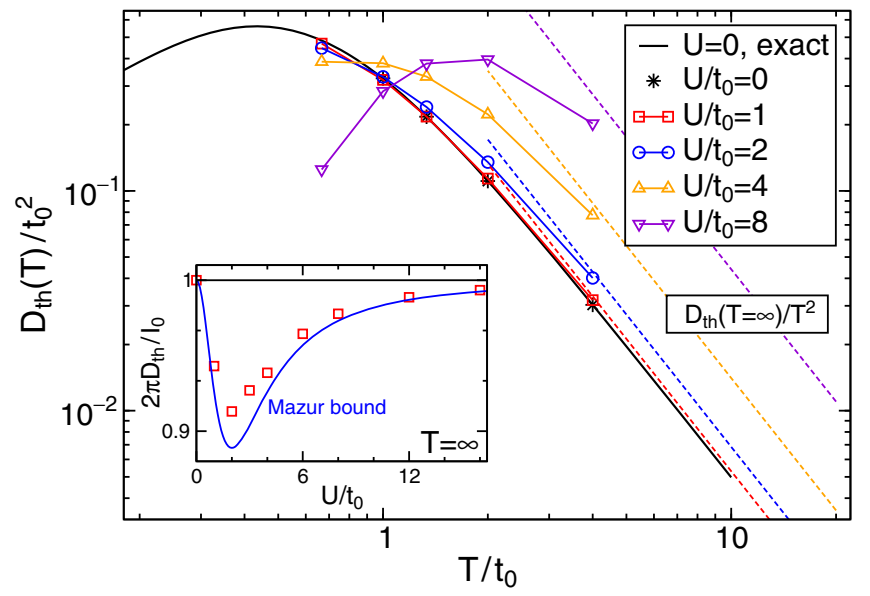

FIG. 2. Thermal Drude weight of the integrable model versus the temperature for $U / t_{0}=0,1,2,4,8$. Solid line: Exact result Eq. (6) for $U=0$, in excellent agreement with DMRG data. Dashed lines: High- $T$ behavior $D_{\text {th }}=D_{\text {th }}^{\infty} / T^{2}$, with $D_{\text {th }}^{\infty}$ computed using DMRG. Inset: $2 \pi D_{\text {th }} / I_{0}$ at $T=\infty$ (squares), compared to the lower Mazur bound [9] (solid line). exact conservation of the thermal current in the noninteracting case, while, in the latter case, it is a consequence of a full suppression of any scattering between subspaces with different numbers of doublons as $U / t_{0}$ diverges. For a finite $0<U / t_{0}<\infty, 2 \pi D_{\text {th }} / I_{0}<1$, and it takes a minimum with $2 \pi D_{\text {th }} / I_{0} \approx 0.92$ close to $U=2 t_{0}$, implying that at $n=1$, the dominant contribution to $\operatorname{Re} \kappa(\omega)$ always comes from the Drude weight. Reference [9] provides a nonzero lower bound for $D_{\text {th }}$ at $T=\infty$ by considering only $Q_{3}$ (a close relative of $I_{\text {th }}$ [34]) in Eq. (2). By comparison to this lower bound (solid line in the inset in Fig. 2), we conclude that the position of this minimum can be understood from the competition of the $U$-dependent and $U$-independent contributions to the Drude weight and to the total weight $I_{0} \propto\left\langle I_{\mathrm{th}}^{2}\right\rangle$. Moreover, the lower bound from Ref. [9] is not exhaustive (see also Fig. S2 in Ref. [34] showing $D_{\text {th }}$ and the lower bound as a function of $n$ ).

Nonintegrable model and low-frequency dependence of $\kappa_{\text {reg }}(\omega)$.- Upon breaking integrability, our results for $C_{\text {th }}(t)$ indicate a vanishing Drude weight, at least at high temperatures and for intermediate values of $U / t_{0}$. This raises the question of the functional form of $\kappa_{\text {reg }}(\omega)$ for $V \neq 0$. Figure 3 shows $\kappa_{\text {reg }}(\omega)$ for $U / t_{0}=4$ at $T=\infty$ and $V=U / 3$ (main panel) and $V=0$ (inset). In the nonintegrable case, $\kappa_{\text {reg }}(\omega)$ has a broad peak at zero frequency, which is very close to a Lorentzian (the dashed line is a fit to the data). This demonstrates that standard diffusion is realized in the extended Hubbard model. In the integrable case, we often observe maxima in $\kappa_{\text {reg }}(\omega)$ at $\omega>0$, which seem to be related to the charge gap. Because of the uncertainties involved in extracting the frequency dependence, which are due to the finite times reached in the simulations and the extraction of the Drude weight, we are not able to resolve the low-frequency regime for $V=0$. Therefore, the question of whether $\kappa_{\text {reg }}(\omega \rightarrow 0)$ is zero or finite in the integrable case, which has been intensely studied for spin transport in the spin-1/2 XXZ chain [19,53,95-98], remains an open problem.

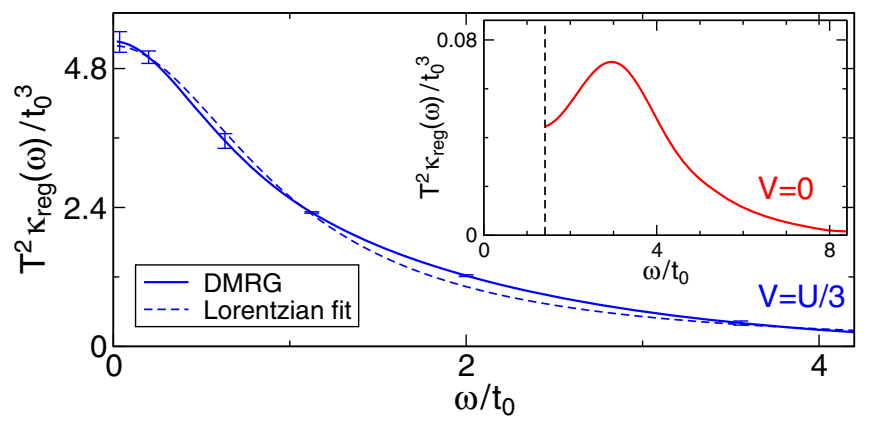

FIG. 3. Regular part $\kappa_{\text {reg }}(\omega)$ for $U / t_{0}=4$ at $T=\infty$. Main panel: Nonintegrable case $(V=U / 3)$. Dashed line: Fit of DMRG data to a Lorentzian. Inset: Integrable case $(V=0)$. The data are shown only for $\omega / t_{0} \gtrsim 1.4$, whereas for smaller frequencies uncertainties become too large (see the text). 

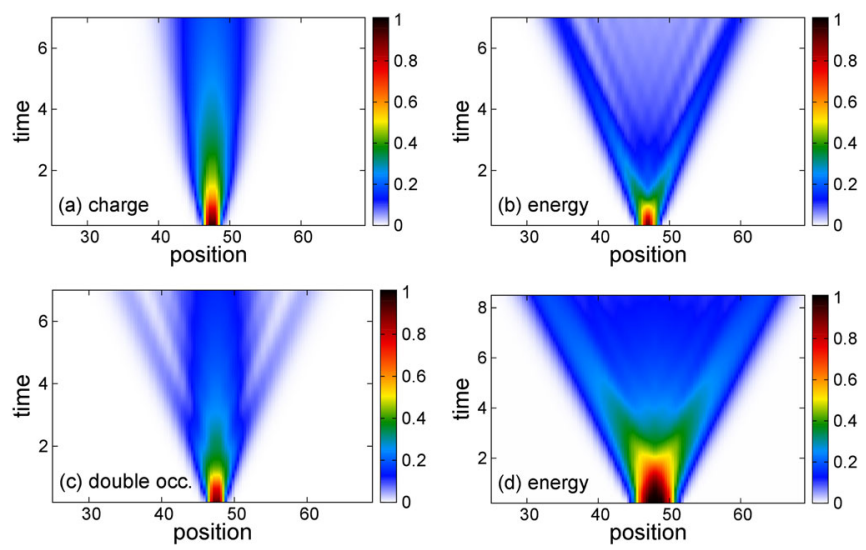

FIG. 4. Local quenches for $U / t_{0}=8$ and $V=0$. (a)-(c) At time $t=0$, a local excitation $(|0\rangle+|\uparrow\rangle+|\downarrow\rangle+1.1|\uparrow \downarrow\rangle)$ is prepared at two sites in the center of an otherwise equilibrated system at temperature $T=\infty$. Real-time evolution of (a) the normalized local charge density $\left[\left\langle n_{l}(t)\right\rangle-1\right] / n_{0}$, (b) the normalized local energy density $\left\langle h_{l}(t)\right\rangle / E_{0}$, and (c) the relative probability for double occupancy $\left[P\left(n_{l}=2\right)-1 / 4\right] / p_{0}$. The normalization constants read $n_{0}=\left(2 \times 1.1^{2}+2\right) /\left(1.1^{2}+3\right)-1$, $E_{0} \approx 0.025 t_{0}$, and $p_{0}=1.1^{2} /\left(1.1^{2}+3\right)-1 / 4$. (d) $T$ quench $\left(T_{1} / t_{0}=10, T_{2}=\infty\right)$ : relative energy density $\left\langle h_{l}(t)\right\rangle /\left(-0.245 t_{0}\right)$.

Spreading of local perturbations.-The presence of a ballistic contribution in the linear response functions translates into the ballistic spreading of perturbations in the local density [18,21,99-103]. To illustrate this connection, we study a Hubbard chain at an infinite temperature and introduce a perturbation in the local charge density at $t=0^{+}$. This also causes a perturbation in the energy density. We measure the time evolution of both densities $\rho_{\text {ch }, l}(t)=\left\langle n_{l}(t)\right\rangle$ and $\rho_{\mathrm{th}, l}(t)=\left\langle h_{l}(t)\right\rangle$, presented in Figs. 4(a) and 4(b). While the energy density shows the typical features of a ballistic dynamics [21,101], the charge density exhibits a much slower spreading and does not form fast ballistic jets, nicely illustrating the different nature of energy versus charge transport in this model. To become more quantitative, we compute the spatial variances associated with the density $\rho_{\mathrm{th}, \mathrm{ch}, l}(t)$ :

$$
\sigma_{\mathrm{th}, \mathrm{ch}}^{2}(t)=\frac{1}{\mathcal{N}_{\mathrm{th}, \mathrm{ch}}} \sum_{l=n_{0}}^{L-n_{0}}\left(l-l_{0}\right)^{2}\left[\rho_{\mathrm{th}, \mathrm{ch}, l}(t)-\rho_{\mathrm{th}, \mathrm{ch}}^{\mathrm{bg}}\right],
$$

where $l_{0}$ is the center of the wave packet, $n_{0}$ cuts off boundary effects, $\rho_{\text {th,ch, } l}^{\text {bg }}$ denotes the bulk background density, and $\mathcal{N}_{\text {th,ch }}$ is the excess particle number or energy induced by the wave packet. As expected, we find $\delta \sigma_{\text {th }}^{2}=$ $\sigma_{\mathrm{th}}^{2}(t)-\sigma_{\mathrm{th}}^{2}(t=0) \propto t^{2}$ yet a much slower growth for the charge $\sigma_{\mathrm{ch}}^{2} \propto t^{\alpha}$ with $1 / 2<\alpha<1$ (see Fig. S4). The determination of the exact exponent would require longer times and is related to the low-frequency behavior of the charge conductivity, yet, clearly, charge dynamics is not ballistic. Another illustration for the ballistic energy spreading can be obtained in $T$ quenches [21], in which we embed a region with $T_{2}$ into a larger system that is at $T_{1}<T_{2}$, which overall has a homogeneous spin and charge density. An example is shown in Fig. 4(d), and, as expected, the variance of this wave packet grows as $\delta \sigma_{\mathrm{th}}(t)^{2} \propto t^{2}$, illustrating that energy spreads ballistically in the temperature quench as well.

The time evolution of the double occupancy (accessible in optical-lattice experiments) $d(t)=\left\langle n_{i \uparrow} n_{i \downarrow}\right\rangle(t)$ is shown in Fig. 4(c) for the quench of Figs. 4(a) and 4(b). The profile exhibits fast ballistic jets, and the associated variance $\sigma_{d}^{2}(t)=1 / D \sum_{l}\left(l-l_{0}\right)^{2}\left[\left\langle d_{l}(t)\right\rangle-d^{\mathrm{bg}}\right] \quad\left(D=\sum_{l}\left\langle d_{l}\right\rangle\right)$ increases approximately quadratically at long times and is thus sensitive to the fast spreading of the energy density. For $V \neq 0$ (see Fig. S5 for an illustration), the variances of both energy and double occupancy increase much slower than quadratically in time. As a consequence of the ballistic energy transport in a 1D FHM, we expect the absence of thermalization in related quantum-gas experiments. From the long-time behavior of the respective width $\delta \sigma_{\mathrm{th}, \mathrm{ch}}(t)$, one can extract diffusion constants [18,21] or Drude weights (see, e.g., [103]) via Einstein relations (as we verified for our case), providing an experimental means of measuring transport coefficients. Such a local real-space and real-time probe for thermal transport has recently been used in experiments with low-dimensional quantum magnets [104]. Given that a coupling to phonons cannot be avoided in quantum magnets [105,106], quantum-gas microscope experiments [58-64] could provide a means of studying energy and charge transport in the FHM, which is easier to realize with ultracold quantum gases than the spin- $1 / 2 X X Z$ chain in its massive regime, where a similar coexistence of diffusive spin transport $[20,21]$ and ballistic energy transport exists $[9,11,107]$.

Summary and outlook.-We computed the thermal conductivity of the 1D FHM using a finite- $T$ DMRG method. We confirm the ballistic nature of thermal transport in the integrable case, and we studied the temperature dependence of the Drude weight. The lower bound for $D_{\text {th }}$ from Ref. [9] is not exhaustive, implying that more local (or even quasilocal $[12,15,33])$ conserved quantities than just $Q_{3}$ play a role. We further demonstrated that the coexistence of diffusive charge transport and ballistic thermal transport is directly reflected in local quantum quench dynamics, presumably accessible to fermionic quantumgas microscopes [58-64]. For the extended Hubbard model, we identified regimes in which, first, the Drude weight clearly vanishes as system size increases and, second, the low-frequency dependence is compatible with diffusive dynamics.

From the theoretical point of view, an exact calculation of $\kappa(\omega)$ exploiting the integrability of the model constitutes an open problem. In view of the existence of quasi-1D materials described by the (extended) Hubbard model (including some Beechgard salts [108,109], organic materials [110-112], and anorganic systems such as $\mathrm{Sr}_{2} \mathrm{CuO}_{3}$ 
[113] and carbon nanotubes [114-116]), a detailed analysis of energy transport and the calculation of diffusion constants is desirable. Finally, the investigation of thermoelectric effects in SCSs is a timely topic (see, e.g., [45,117121]) and should be feasible with our technique, at least at high temperatures.

We thank C. Hess, T. Prosen, R. Steinigeweg, and X. Zotos for very helpful discussions. We thank M. Medenjak and T. Prosen for pointing out a mistake in a previous version of Fig. S2 to us. We acknowledge support by the Nanostructured Thermoelectrics program of LBNL (C. K.) as well as by the DFG through the Research Training Group 1995 (D. M. K.) and the Emmy Noether program (C. K.).

[1] S. R. White, Phys. Rev. Lett. 69, 2863 (1992).

[2] U. Schollwöck, Rev. Mod. Phys. 77, 259 (2005).

[3] U. Schollwöck, Ann. Phys. (Berlin) 326, 96 (2011).

[4] F. Essler, H. Frahm, F. Göhmann, A. Klümper, and V. E. V. E. Korepin, The One-Dimensional Hubbard Model (Cambridge University Press, Cambridge, England, 2005).

[5] T. Giamarchi, Quantum Physics in One Dimension (Clarendon, Oxford, 2004), p. 2905.

[6] A. Klümper and D. C. Johnston, Phys. Rev. Lett. 84, 4701 (2000).

[7] J.-S. Caux and J. M. Maillet, Phys. Rev. Lett. 95, 077201 (2005).

[8] R. G. Pereira, J. Sirker, J.-S. Caux, R. Hagemans, J. M. Maillet, S. R. White, and I. Affleck, Phys. Rev. Lett. 96, 257202 (2006).

[9] X. Zotos, F. Naef, and P. Prelovšek, Phys. Rev. B 55, 11029 (1997).

[10] A. Klümper and K. Sakai, J. Phys. A 35, 2173 (2002).

[11] K. Sakai and A. Klümper, J. Phys. A 36, 11617 (2003).

[12] T. Prosen, Phys. Rev. Lett. 106, 217206 (2011).

[13] J. Herbrych, P. Prelovšek, and X. Zotos, Phys. Rev. B 84, 155125 (2011).

[14] C. Karrasch, J. Hauschild, S. Langer, and F. HeidrichMeisner, Phys. Rev. B 87, 245128 (2013).

[15] T. Prosen and E. Ilievski, Phys. Rev. Lett. 111, 057203 (2013).

[16] R. Steinigeweg, J. Gemmer, and W. Brenig, Phys. Rev. Lett. 112, 120601 (2014).

[17] P. Prelovšek, S. ElShawish, X. Zotos, and M. W. Long, Phys. Rev. B 70, 205129 (2004).

[18] R. Steinigeweg and J. Gemmer, Phys. Rev. B 80, 184402 (2009).

[19] J. Sirker, R. G. Pereira, and I. Affleck, Phys. Rev. B 83, 035115 (2011).

[20] R. Steinigeweg and W. Brenig, Phys. Rev. Lett. 107, 250602 (2011).

[21] C. Karrasch, J. E. Moore, and F. Heidrich-Meisner, Phys. Rev. B 89, 075139 (2014).

[22] S. Kirchner, H. G. Evertz, and W. Hanke, Phys. Rev. B 59, 1825 (1999).

[23] N. M. R. Peres, R. G. Dias, P. D. Sacramento, and J. M. P. Carmelo, Phys. Rev. B 61, 5169 (2000).
[24] J. Carmelo, S.-J. Gu, and P. Sacramento, Ann. Phys. (Berlin) 339, 484 (2013).

[25] T. Prosen and M. Žnidarič, Phys. Rev. B 86, 125118 (2012).

[26] T. Prosen, Phys. Rev. Lett. 112, 030603 (2014).

[27] C. Karrasch, D. M. Kennes, and J. E. Moore, Phys. Rev. B 90, 155104 (2014).

[28] F. Jin, R. Steinigeweg, F. Heidrich-Meisner, K. Michielsen, and H. De Raedt, Phys. Rev. B 92, 205103 (2015).

[29] G. D. Mahan, Many-Particle Physics (Plenum, New York, 1990).

[30] J. M. Luttinger, Phys. Rev. 135, A1505 (1964).

[31] M. Suzuki, Physica (Amsterdam) 51, 277 (1971).

[32] P. Mazur, Physica (Amsterdam) 43, 533 (1969).

[33] M. Mierzejewski, P. Prelovšek, and T. Prosen, Phys. Rev. Lett. 114, 140601 (2015)

[34] See Supplemental Material at http://link.aps.org/ supplemental/10.1103/PhysRevLett.117.116401 for definitions, a discussion of transport coefficients, DMRG data for several system sizes, a comparison of the thermal Drude weight of the FHM to the one of the Heisenberg chain, and additional results (Figs. S4 and S5) for local quenches, including Refs. [35-46].

[35] N. W. Ashcroft and N. D. Mermin, Solid State Physics (Saunders, Philadelphia, 1976).

[36] R. Steinigeweg (private communication).

[37] D. Karevski and T. Platini, Phys. Rev. Lett. 102, 207207 (2009).

[38] T. Prosen and B. Zunkovic, New J. Phys. 12, 025016 (2010).

[39] M. Žnidarič, Phys. Rev. Lett. 106, 220601 (2011).

[40] J. J. Mendoza-Arenas, T. Grujic, D. Jaksch, and S. R. Clark, Phys. Rev. B 87, 235130 (2013).

[41] A. V. Sologubenko, K. Gianno, H. R. Ott, U. Ammerahl, and A. Revcolevschi, Phys. Rev. Lett. 84, 2714 (2000).

[42] C. Hess, C. Baumann, U. Ammerahl, B. Büchner, F. Heidrich-Meisner, W. Brenig, and A. Revcolevschi, Phys. Rev. B 64, 184305 (2001).

[43] A. E. Feiguin and G. A. Fiete, Phys. Rev. Lett. 106, 146401 (2011).

[44] M. Soltanieh-ha and A.E. Feiguin, Phys. Rev. B 90, 165145 (2014).

[45] M. R. Petersen, S. Mukerjee, B. S. Shastry, and J. O. Haerter, Phys. Rev. B 76, 125110 (2007).

[46] C. Hess, Dissertation, Universität zu Köln (2002)

[47] C. Karrasch, J. Bardarson, and J.E. Moore, Phys. Rev. Lett. 108, 227206 (2012).

[48] C. Karrasch, J. H. Bardarson, and J.E. Moore, New J. Phys. 15, 083031 (2013).

[49] T. Barthel, New J. Phys. 15, 073010 (2013).

[50] F. Verstraete, J. J. García-Ripoll, and J. I. Cirac, Phys. Rev. Lett. 93, 207204 (2004).

[51] A. E. Feiguin and S. R. White, Phys. Rev. B 72, 220401 (2005).

[52] A. De Luca, J. Viti, L. Mazza, and D. Rossini, Phys. Rev. B 90, 161101 (2014).

[53] C. Karrasch, D. M. Kennes, and F. Heidrich-Meisner, Phys. Rev. B 91, 115130 (2015).

[54] T. Barthel, U. Schollwöck, and S. R. White, Phys. Rev. B 79, 245101 (2009).

[55] U. Schneider, L. Hackermüller, S. Will, T. Best, I. Bloch, T. A. Costi, R. W. Helmes, D. Rasch, and A. Rosch, Science 322, 1520 (2008). 
[56] R. Jördens, N. Strohmaier, K. Günter, H. Moritz, and T. Esslinger, Nature (London) 455, 204 (2008).

[57] R. A. Hart, P. M. Duarte, T.-L. Yang, X. Liu, T. Paiva, E. Khatami, R. T. Scalettar, N. Trivedi, D. A. Huse, and R. G. Hulet, Nature (London) 519, 211 (2015).

[58] G. J. A. Edge, R. Anderson, D. Jervis, D. C. McKay, R. Day, S. Trotzky, and J. H. Thywissen, Phys. Rev. A 92, 063406 (2015).

[59] A. Omran, M. Boll, T. A. Hilker, K. Kleinlein, G. Salomon, I. Bloch, and C. Gross, Phys. Rev. Lett. 115, 263001 (2015).

[60] E. Haller, J. Hudson, A. Kelly, D. Cotta, B. Peaudecerf, G. Bruce, and S. Kuhr, Nat. Phys. 11, 738 (2015).

[61] D. Greif, M. F. Parsons, A. Mazurenko, C. S. Chiu, S. Blatt, F. Huber, G. Ji, and M. Greiner, Science 351, 953 (2016).

[62] M. Boll, T. A. Hilker, G. Salomon, A. Omran, I. Bloch, and C. Gross, arXiv:1605.05661.

[63] E. Cocchi, L. A. Miller, J. H. Drewes, M. Koschorreck, D. Pertot, F. Brennecke, and M. Köhl, Phys. Rev. Lett. 116, 175301 (2016).

[64] L. W. Cheuk, M. A. Nichols, K. R. Lawrence, M. Okan, H. Zhang, and M. W. Zwierlein, Phys. Rev. Lett. 116, 235301 (2016).

[65] C.-L. Hung, X. Zhang, N. Gemelke, and C. Chin, Phys. Rev. Lett. 104, 160403 (2010).

[66] B. Schmidt, M. R. Bakhtiari, I. Titvinidze, U. Schneider, M. Snoek, and W. Hofstetter, Phys. Rev. Lett. 110, 075302 (2013).

[67] D. McKay and B. DeMarco, Rep. Prog. Phys. 74, 054401 (2011).

[68] U. Schneider, L. Hackermüller, J. P. Ronzheimer, S. Will, S. Braun, T. Best, I. Bloch, E. Demler, S. Mandt, D. Rasch, and A. Rosch, Nat. Phys. 8, 213 (2012).

[69] S. Trotzky, Y.-A. Chen, A. Flesch, I. P. McCulloch, U. Schollwöck, J. Eisert, and I. Bloch, Nat. Phys. 8, 325 (2012).

[70] J. Ronzheimer, M. Schreiber, S. Braun, S. Hodgman, S. Langer, I. McCulloch, F. Heidrich-Meisner, I. Bloch, and U. Schneider, Phys. Rev. Lett. 110, 205301 (2013).

[71] D. Pertot, A. Sheikhan, E. Cocchi, L. A. Miller, J. E. Bohn, M. Koschorreck, M. Köhl, and C. Kollath, Phys. Rev. Lett. 113, 170403 (2014).

[72] G. Beni and C. F. Coll, Phys. Rev. B 11, 573 (1975).

[73] R. Kubo, J. Phys. Soc. Jpn. 12, 570 (1957).

[74] R. Kubo, M. Yokota, and S. Nakajima, J. Phys. Soc. Jpn. 12, 1203 (1957).

[75] J. M. Luttinger, Phys. Rev. 135, A1505 (1964).

[76] K. Louis and C. Gros, Phys. Rev. B 67, 224410 (2003).

[77] J. Gemmer, R. Steinigeweg, and M. Michel, Phys. Rev. B 73, 104302 (2006).

[78] M. Michel, G. Mahler, and J. Gemmer, Phys. Rev. Lett. 95, 180602 (2005).

[79] T. Prosen and M. Žnidarič, J. Stat. Mech. (2009) P02035.

[80] L. Arrachea, G. S. Lozano, and A. A. Aligia, Phys. Rev. B 80, 014425 (2009).

[81] J. J. Mendoza-Arenas, S. Al-Assam, S. R. Clark, and D. Jaksch, J. Stat. Mech. (2013) P07007.

[82] M. Fannes, B. Nachtergaele, and R. F. Werner, J. Phys. A 24, L185 (1991).

[83] S. Östlund and S. Rommer, Phys. Rev. Lett. 75, 3537 (1995).
[84] F. Verstraete and J. I. Cirac, Phys. Rev. B 73, 094423 (2006).

[85] F. Verstraete, J. I. Cirac, and V. Murg, Adv. Phys. 57, 143 (2008).

[86] F. Verstraete, J. J. Garcia-Ripoll, and J. I. Cirac, Phys. Rev. Lett. 93, 207204 (2004).

[87] S. White, Phys. Rev. Lett. 102, 190601 (2009).

[88] M. Zwolak and G. Vidal, Phys. Rev. Lett. 93, 207205 (2004).

[89] J. Sirker and A. Klümper, Phys. Rev. B 71, 241101(R) (2005).

[90] E. Jeckelmann, Phys. Rev. Lett. 89, 236401 (2002).

[91] X. Zotos and P. Prelovšek, Phys. Rev. B 53, 983 (1996).

[92] F. Heidrich-Meisner, A. Honecker, D. C. Cabra, and W. Brenig, Phys. Rev. B 68, 134436 (2003).

[93] X. Zotos, Phys. Rev. Lett. 92, 067202 (2004).

[94] P. Jung, R. W. Helmes, and A. Rosch, Phys. Rev. Lett. 96, 067202 (2006).

[95] J. Sirker, R. G. Pereira, and I. Affleck, Phys. Rev. Lett. 103, 216602 (2009).

[96] S. Grossjohann and W. Brenig, Phys. Rev. B 81, 012404 (2010).

[97] J. Herbrych, R. Steinigeweg, and P. Prelovšek, Phys. Rev. B 86, 115106 (2012).

[98] Y. Rahnavard, R. Steinigeweg, and W. Brenig, arXiv: 1501.03447.

[99] M. Polini and G. Vignale, Phys. Rev. Lett. 98, 266403 (2007).

[100] S. Langer, F. Heidrich-Meisner, J. Gemmer, I. McCulloch, and U. Schollwöck, Phys. Rev. B 79, 214409 (2009).

[101] S. Langer, M. Heyl, I. P. McCulloch, and F. HeidrichMeisner, Phys. Rev. B 84, 205115 (2011).

[102] S. Jesenko and M. Žnidarič, Phys. Rev. B 84, 174438 (2011).

[103] Y. Yan, F. Jiang, and H. Zhao, Eur. Phys. J. B 88, 11 (2015).

[104] M. Otter, V. Krasnikov, D. Fishman, M. Pshenichnikov, R. Saint-Martin, A. Revcolevschi, and P. van Loodsrecht, J. Magn. Magn. Mater. 321, 796 (2009).

[105] C. Hess, Eur. Phys. J. Spec. Top. 151, 73 (2007).

[106] M. Montagnese, M. Otter, X. Zotos, D. A. Fishman, N. Hlubek, O. Mityashkin, C. Hess, R. Saint-Martin, S. Singh, A. Revcolevschi, and P. H. M. van Loosdrecht, Phys. Rev. Lett. 110, 147206 (2013).

[107] F. Heidrich-Meisner, A. Honecker, D. C. Cabra, and W. Brenig, Phys. Rev. B 66, 140406 (2002).

[108] D. Jerome, Chem. Rev. 104, 5565 (2004).

[109] V. Vescoli, L. Degiorgi, W. Henderson, G. Grüner, K. P. Starkey, and L. K. Montgomery, Science 281, 1181 (1998).

[110] T. Hasegawa, S. Kagoshima, T. Mochida, S. Sugiura, and Y. Iwasa, Solid State Commun. 103, 489 (1997).

[111] R. Claessen, M. Sing, U. Schwingenschlögl, P. Blaha, M. Dressel, and C. S. Jacobsen, Phys. Rev. Lett. 88, 096402 (2002).

[112] S. Wall, D. Brida, S. R. Clark, H. P. Ehrke, D. Jaksch, A. Ardavan, S. Bonora, H. Uemura, Y. Takahashi, T. Hasegawa, H. Okamoto, G. Cerullo, and A. Cavalleri, Nat. Phys. 7, 114 (2011).

[113] M. Ono, K. Miura, A. Maeda, H. Matsuzaki, H. Kishida, Y. Taguchi, Y. Tokura, M. Yamashita, and H. Okamoto, Phys. Rev. B 70, 085101 (2004). 
[114] M. Bockrath, D. H. Cobden, J. Lu, A. G. Rinzler, R. E. Smalley, L. Balents, and P. L. McEuen, Nature (London) 397, 598 (1999).

[115] H. Ishii, H. Kataura, H. Shiozawa, H. Yoshioka, H. Otsubo, Y. Takayama, T. Miyahara, S. Suzuki, Y. Achiba, M. Nakatake, T. Narimura, M. Higashiguchi, K. Shimada, H. Namatame, and M. Taniguchi, Nature (London) 426, 540 (2003).

[116] V. V. Deshpande, B. Chandra, R. Caldwell, D. Novikov, J. Hone, and M. Bockrath, Science 323, 106 (2009).
[117] B. Shastry, Rep. Prog. Phys. 72, 016501 (2009).

[118] P. Wissgott, A. Toschi, H. Usui, K. Kuroki, and K. Held, Phys. Rev. B 82, 201106 (2010).

[119] S. Hong, P. Ghaemi, J. E. Moore, and P. W. Phillips, Phys. Rev. B 88, 075118 (2013).

[120] M. Mierzejewski, D. Crivelli, and P. Prelovšek, Phys. Rev. B 90, 075124 (2014).

[121] J. Kokalj and R. H. McKenzie, Phys. Rev. B 91, 125143 (2015). 